\title{
Élever des poissons pour nourrir la planète
}

\section{Guy Paillotin}

\section{Secrétaire perpétuel}

de l'Académie d'agriculture de France

Président du comité scientifique

des Cahiers Agricultures

Académie d'agriculture de France

18 , rue de Bellechasse

75007 Paris

<corinne.migne@paris.inra.fr>
C e numéro des Cabiers Agricultures doit être dédié à René Lésel. Après sept ans de services inestimables rendus à notre revue comme rédacteur en chef, il a décidé, à notre grand regret, de passer la main, parce qu'il estime le moment venu et qu'il souhaite s'investir autrement dans sa vie personnelle. Le rythme de notre programmation fait que ce dernier numéro, publié sous sa direction, est consacré à la pisciculture. Chacun sait bien qu'avec son épouse, il a consacré sa vie professionnelle, et sans doute plus, à cette importante question. Ils ont trouvé dans leur œuvre de nombreuses satisfactions, des difficultés aussi, mais ils peuvent être fiers du devoir accompli et de la reconnaissance internationale qui est la leur.

Ce numéro a été réalisé à l'initiative de, et en collaboration étroite avec, Jérôme Lazard qui a apporté sa compétence reconnue à l'échelle internationale dans la domestication et l'élevage d'espèces vivant en eaux chaudes. Il a su aussi communiquer dans les textes présentés sa conviction sur la place croissante, dès aujourd'hui, de l'aquaculture comme source de protéines pour les populations humaines.

En effet, la pisciculture joue, et jouera encore plus dans l'avenir, un rôle essentiel dans l'alimentation du monde. On constate aujourd'hui que la misère n'est pas endiguée par les seules vertus de la libéralisation des échanges de denrées. On constate aussi que, à côté de la détresse alimentaire, se développe une insuffisance nutritionnelle qui altère sévèrement le développement des adultes et surtout celui des enfants, dans presque la moitié du monde.

Il est grand temps - et la FAO nous y invite - de nous mobiliser pour faire face à cet enjeu vital pour l'équilibre du monde.
Dans ce contexte, beaucoup de nutritionnistes rappellent que le poisson est un vecteur de l'ensemble des nutriments et sa consommation est de ce fait qualitativement un facteur d'équilibre nutritionnel incontournable. Le calcul rapide du volume des besoins mondiaux fixés par cette exigence nutritionnelle comparé à nos capacités de production, montre que ces deux données sont du même ordre de grandeur. Si tout est mis en œuvre au plan mondial pour assurer la ressource, sa couverture dépend de façon très rigide de la pisciculture.

Ce numéro de nos Cahiers Agricultures rassemble donc les éléments techniques existants ou à venir qui permettraient de relever ce défi. J'ai volontairement utilisé le conditionnel, car l'existence de techniques de production ne suffit pas. Encore faut-il qu'elles diffusent auprès de ceux qui en ont le plus besoin ; encore faut-il aussi qu'ils aient les moyens d'investir pour les mettre en œuvre.

Mais nous ne sommes pas encore au bout de nos peines. Pour faire face aux enjeux nutritionnels que j’ai évoqués, la pisciculture ne peut pas ne pas être relativement intensive. Se pose alors la question de sa durabilité. Comment faire pour ne pas épuiser les ressources génétiques? Comment faire pour ne pas créer de nuisances environnementales préjudiciables, à terme, à la production elle-même ? Comment répartir la richesse que pourrait créer la pisciculture de façon suffisamment harmonieuse pour favoriser l'entreprise sans provoquer de l'exclusion?

Fidèles à ce qui est leur " mission ", nos Cabiers n'éludent aucune de ces questions, car elles doivent toutes être abordées de façon cohérente. Merci donc mille fois aux nombreux auteurs qui ont contribué à ce numéro exceptionnel. 